\title{
Performance Enhancement of
}

\section{FLUORESCENCE ENERGY Transfer-BASED BIOSENSORS BY SITE-DIRECTED MUTAGENESIS OF THE TRANSDUCER}

\author{
Richard B. Thompson, ${ }^{\dagger}$ Zhengfang Ge ${ }^{\dagger}$ Marcia W. Patchan, ${ }^{\dagger}$ and Carol A. Fierke ${ }^{\ddagger}$ \\ ${ }^{\dagger}$ University of Maryland at Baltimore, Department of Biological Chemistry; ${ }^{\dagger}$ Duke University Medical \\ Center, Department of Biochemistry, Durham, North Carolina \\ (Paper JBO-037 received Aug. 25, 1995; accepted for publication Oct. 22, 1995)
}

\begin{abstract}
We have recently described a biosensor for zinc and other metals which is based upon fluorescence energy transfer from a site or sites on the enzyme carbonic anhydrase to an inhibitor whose binding to the active site is largely metal-dependent. The concentration of the metal ion analyte is a simple function of the occupancy of the active site by the colored inhibitor, which is transduced as a change in intensity or lifetime of the fluorescent donor. We and others have demonstrated assays for several analytes based on energy transfer with moderate success. In this work we demonstrate by simulation and experiment that precise control of the donor:acceptor distance is valuable in optimizing the response of the system.
\end{abstract}

Key Words Biosensors; mutagenesis; transducers; fluorometry; fluorescence energy transfer.

\section{INTRODUCTION}

Fluorescence-based biosensors are beginning to find broad applicability to many chemical analysis problems of interest. ${ }^{1-5}$ The move to biosensing has been fueled in part by the requirement for high selectivity in the recognition of analytes such as metal ions, ${ }^{6}$ small molecules, ${ }^{7}$ and macromolecules ${ }^{8}$ in complex natural matrices such as seawater, soil, and blood. While several fluorescence-based transduction approaches have been described, including analyte-induced changes in intensity, 9,10 wavelength, ${ }^{6,11}$ and lifetime, ${ }^{12-14}$ the last named has been found to have several advantages in sensing applications. The advantages of fluorescence lifetime-based sensing include relative freedom from artifacts due to source fluctuations, scattering or colored samples; variation in indicator concentration due to matrix effects, photobleaching, or small changes in solution composition or temperature; facile incorporation into fiber optic systems; wavelength flexibility; facile calibration; a very large dynamic range ( $>5$ decades) in favorable cases, ${ }^{15,16}$ and a large technology base of chemistry, hardware, software, and experience in fluorescence techniques. ${ }^{14,17,18}$ Inasmuch as fluorescence resonance energy transfer results in quenching of the donor fluorophore with a concomitant reduction in the lifetime, this phenomenon has been used for fluorescence lifetime-based determination of several classes of analytes, including ions, ${ }^{19,20}$ small molecules, ${ }^{21}$ and macromolecules by immunoassay. ${ }^{22}$

Address all correspondence to Richard B. Thompson, University of Maryland at Baltimore, Department of Biological Chemistry, 108 N. Greene St., Baltimore, Maryland 21201.
For the sensing of zinc we have employed a biological transducer incorporating the enzyme human carbonic anhydrase (CA) II, which binds $\mathrm{Zn}^{2+}$ tightly $\left(K_{d}=4 \mathrm{pM}\right)$ and relatively specifically in its active site, particularly in comparison with most metallofluorescent indicators. ${ }^{23}$ The transduction scheme is depicted in Figure 1, in which the enzyme is derivatized with some suitable fluorescent label. If zinc is removed from the active site, the colored inhibitor azosulfamide tends not to bind, no energy transfer takes place and the unperturbed lifetime of the label is observed. However, if zinc is present it will occupy the active site to a degree determined by the laws of mass action, and will consequently induce the binding of the azosulfamide, which is thus held in close proximity to the label so that energy transfer will occur. The energy transfer is a net quenching of the label fluorescence, which reduces the lifetime commensurately. Thus as the analyte concentration increases, a greater fraction of the enzyme binding sites become occupied and the proportion of labels exhibiting the short lifetime increases, resulting in a reduced average lifetime.

In some of the foregoing examples of lifetimebased sensing, the response in terms of net perturbation of the lifetime due to saturation of the sensor with the analyte was modest. When using phasemodulation (frequency-domain) fluorometry, ${ }^{24,25}$ we can express the response of the transducer (labeled apoenzyme plus colored inhibitor) as the total change in phase angle or modulation in going from zero to complete occupancy. ${ }^{14}$ Obviously, we would

1083-3668/96/\$6.00 @ 1996 SPIE 


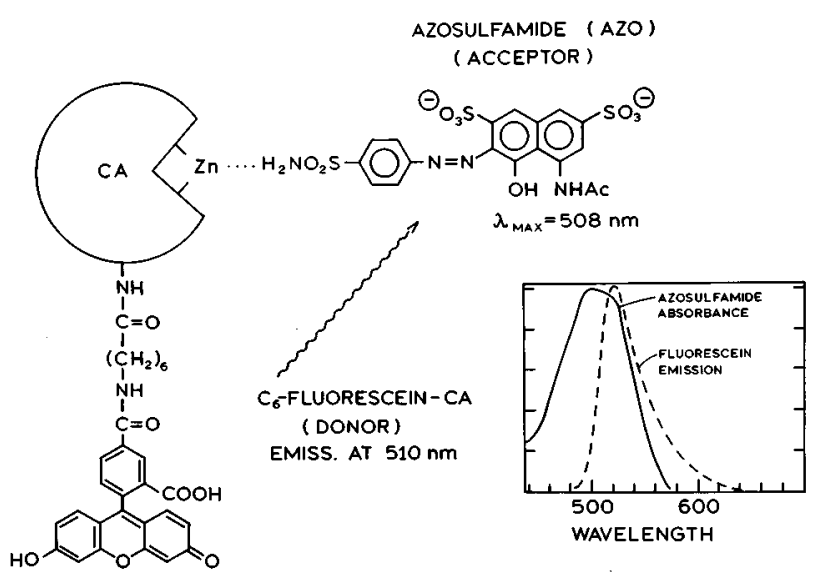

Fig. 1 Principle of energy transfer-based biosensor. In the absence of zinc ion the colored inhibitor azosulfamide does not bind appreciably to fluorescent-labeled apocarbonic anhydrase, no energy transfer occurs, and the fluorescent label (fluorescein, for instance) exhibits its normal, unquenched lifetime. If zinc is added, it binds to the active site and permits azosulfamide to bind, which is now close enough for energy transfer to occur due to the spectral overlap between the fluorescein emission and the azosulfamide absorbance. The energy transfer results in net quenching of the label fluorescence, and a concomitant reduction of the lifetime.

like this change to be as large as possible to improve the accuracy and dynamic range of the sensor. $\mathrm{We}^{16}$ and others ${ }^{15}$ have demonstrated phase angle differences of more than 50 degrees arising from sevenfold changes in lifetime. In these examples the shorter-lived form of the fluorophore did not necessarily have a commensurately reduced quantum yield; that is, the two forms of the fluorescent indicator (analyte-free and bound) did not necessarily have similar intrinsic lifetimes. In the case of energy transfer-based transduction, the bound form is simply a quenched free form, with a commensurately reduced quantum yield. Thus while one could reduce the lifetime manyfold by arranging near-quantitative energy transfer, the corresponding low quantum yield would mean that the quenched, bound form would contribute very little to the total emission except near saturation (see below), and the weak emission might jeopardize collecting accurate data. Of course, if the energy transfer in the bound form is modest, the lifetime change and therefore phase angle and modulation changes will be small as well and less useful. Qualitatively, this suggests that there is some suitable middle ground of energy transfer efficiency that will result in an optimum response where there is a large enough reduction in the lifetime to provide a significant change in the phase angle and modulation (at least 25 degrees and 30\%, respectively) without excessively reducing the quantum yield of the bound form.

We chose to calculate the phase angles and demodulations as a function of zinc concentration for a model system chosen to closely resemble the carbonic anhydrase/Zn/azosulfamide system, where the energy transfer efficiency could be adjusted by changing the distance between donor and acceptor, and making a few simple assumptions. These simulations suggest that positioning the fluorescent label at particular sites on the exterior of carbonic anhydrase should provide an optimum response compared with what we have been able to achieve with nonspecific labeling approaches. ${ }^{19,22}$ Finally, we used site-directed mutagenesis to create carbonic anhydrase variants with cysteine residues at desired points for selective derivatization with suitable labels, to verify that this approach did indeed result in improved response.

\section{THEORY}

Let us assume a system where the metal-free (unquenched) fluorescent-labeled CA exhibits a single exponential decay and the commensurate phase angle at some frequency, and the metal-bound form undergoes energy transfer (and partial quenching) with a concomitant diminution of phase angle at the same frequency. The phase angle we observe as the fraction bound is varied is not simply some weighted sum of those phase angles. Rather, it is somewhat more complex. If the emission of the donor is monoexponential, the time-dependent emission takes the form:

$$
I_{D}(t)=\alpha_{D} e^{\left(t / \tau_{D}\right)}
$$

where $\tau_{D}$ is the lifetime of the donor in the absence of an acceptor. In the presence of the acceptor, there is an additional nonemissive decay process, the energy transfer, which results in a reduced apparent lifetime $\tau_{D A}$ :

$$
\tau_{D A}=\tau_{D}\left[R^{6} /\left(R^{6}+R_{0}^{6}\right)\right],
$$

where $R$ is the distance from donor to acceptor and $R_{0}$ is the well-known "Forster distance," defined as the distance where energy transfer is $50 \%$ efficient (e.g., $\tau_{D A}=\tau_{D} / 2$ ). The Forster distance is in turn primarily a function of the spectral overlap integral between the donor emission and the acceptor absorbance, with other contributions due to the relative orientation of the transition dipoles of the donor and acceptor, and the refractive index of the medium. The rate of energy transfer is a linear function of the overlap integral, but also is inversely proportional to the sixth power of the distance. If we assume that the distance between the donor and acceptor is the same for all donor:acceptor pairs and does not change during the donor excited state, and furthermore that relative orientation between donor and acceptor transition dipoles changes rapidly compared with the time scale of the emission, we can describe the time-dependent emission resulting from the two decay processes:

$$
I_{D A}(t)=\alpha_{D} \exp \left[-\frac{t}{\tau_{D}}-\frac{t}{\tau_{D}}\left(\frac{R_{0}}{R}\right)^{6}\right]
$$


Note that if there is a distribution of distances between donors and acceptors, or if they translate during the lifetime of the donor, these circumstances can be recognized and quantified from the frequency domain data at the current state of the art. In our fairly rigid system we expect that any distance fluctuations will be minor in the holoprotein. The phases and modulations can be calculated from the sine and cosine transforms $\left(N_{\omega}\right.$ and $D_{\omega}$, respectively) of the impulse responses:

$$
\begin{aligned}
N_{\omega} & =\int_{0}^{\infty} I(t) \sin \omega t d t / \int_{0}^{\infty} I(t) d t \\
& =\sum_{i} \frac{\alpha_{i} \omega \tau_{i}^{2}}{\left(1+\omega^{2} \tau_{i}^{2}\right)} / \sum_{i} \alpha_{i} \tau_{i} \\
D_{\omega} & =\int_{0}^{\infty} I(t) \cos \omega t d t / \int_{0}^{\infty} I(t) d t \\
& =\sum_{i} \frac{\alpha_{i} \tau_{i}}{\left(1+\omega^{2} \tau_{i}^{2}\right)} / \sum_{i} \alpha_{i} \tau_{i}
\end{aligned}
$$

$$
\begin{gathered}
\phi_{\omega}=\arctan \left(N_{\omega} / D_{\omega}\right) \\
M_{\omega}=\sqrt{N_{\omega}^{2}+D_{\omega}^{2}},
\end{gathered}
$$

where $\omega$ is the modulation frequency.

We can use these equations to simulate the response of our biosensor transducer. For instance, suppose we have a variant of CA II (such as H36C) where a fluorescein is positioned 30 angstroms from the azosulfamide bound to the zinc (see Figure 2). The Forster distance (where the energy transfer rate equals the emissive rate, and thus the donor lifetime is halved) for the fluorescein:azosulfamide donor:acceptor pair is 40 angstroms, ${ }^{19}$ or perhaps a little less since there seems to be a slight blue shift of the azosulfamide when it is bound to the enzyme. ${ }^{26}$ Thus the apoenzyme exhibits the typical 4-nsec lifetime of fluorescein, whereas the holoprotein with azosulfamide bound is about $85 \%$ quenched (due to the $R^{6}$ dependence of the Forster transfer) and exhibits about a 600-psec lifetime. The

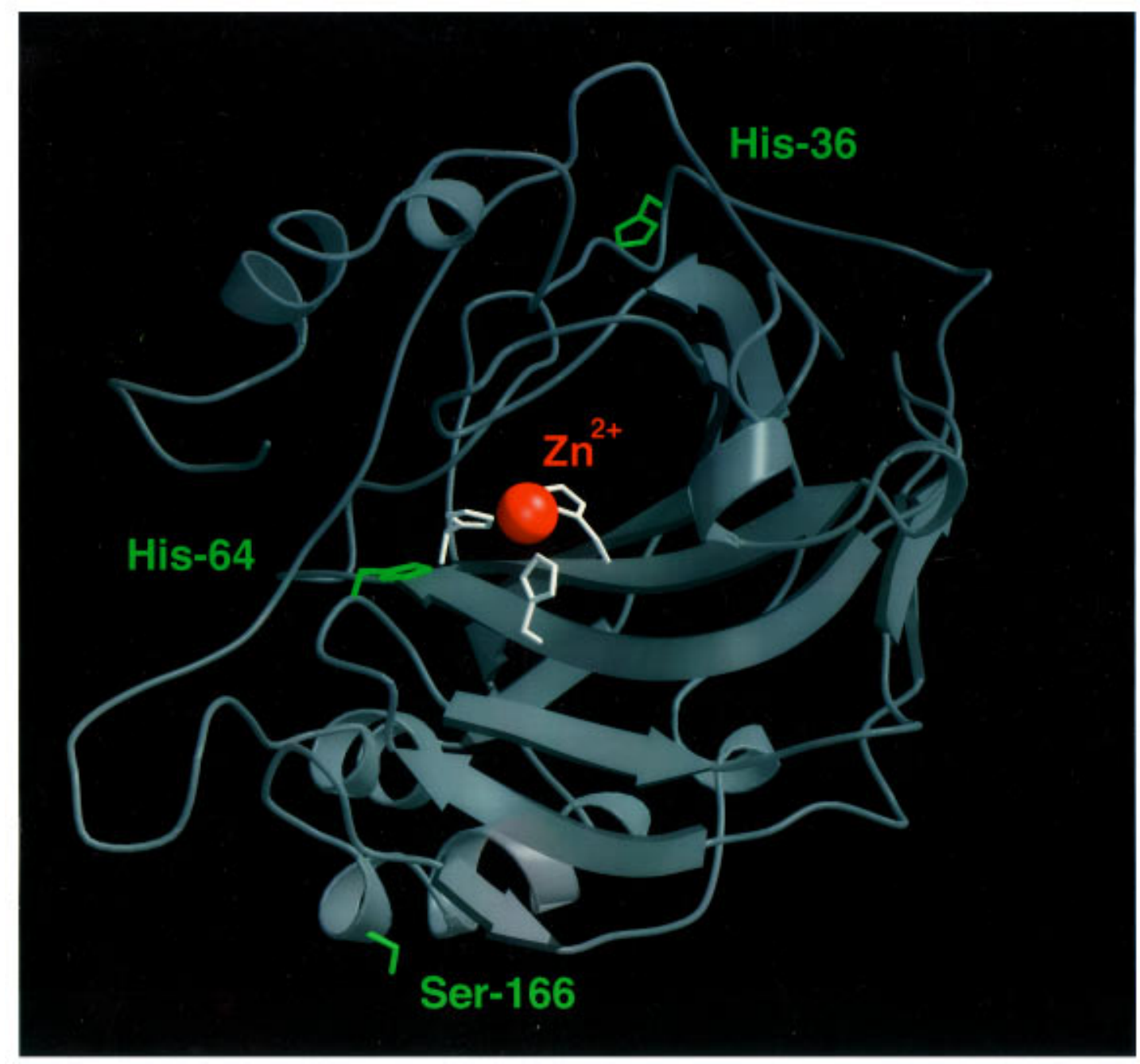

Fig. 2 Human carbonic anhydrase II, showing positions with respect to the active site zinc ion of site-directed mutations at positions 166 (S166C, 22 angstroms from sulfonamide), 64 (H64C, 9 angstroms), and 36 (H36C, 30 angstroms). Image courtesy of C. Lesburg and D. Christianson, University of Pennsylvania. 
simulated $^{27}$ frequency-dependent phase shifts and demodulations for this variant at different fractional saturations of zinc (and thus azosulfamide) are depicted in Figure 3.

If we plot the phases and modulations at some suitable frequency as a function of the free analyte concentration (normalized to $K_{d}$ ) on a logarithmic scale, they appear as Figures 4 and 5, respectively. Note that the upper (nonlinear) scale indicates the fractional saturation. The range of phase angle and modulation in this case is 45 degrees and $50 \%$, respectively. If we move the donor farther away to $R=40$ angstroms (e.g., $R / R_{0}=1$ ), the response is reduced: the phase angle span is now about 25 degrees at best, since the lifetime is reduced by only half when the inhibitor is bound (see Figures 4 and 5). Except under circumstances where the emission is very weak, this position of the donor appears suboptimal compared with the first case $\left(R / R_{0}\right.$ $=0.75$ ). If the donor is positioned much closer to the acceptor $\left(R=22\right.$ angstroms; $R_{0}=40$ angstroms; $R / R_{0}=0.55$ ), we get an even larger phase and modulation change (Figures 4 and 5), but the relative intensity of the quenched form is so small that the changes in phase angle and modulation with $\mathrm{Zn}$ concentration are most pronounced above $90 \%$ saturation. In this case the bound form has a lifetime of about 100 psec and a $2 \%$ quantum yield relative to the unquenched donor. While in principle one can accurately quantitate much higher levels of saturation (e.g., going from 97 to $98 \%$ saturation results in a 5-degree phase shift), in practice getting good data at such low light levels is difficult, due to the (relatively) enhanced contributions of fluorescent impurities.

Based on these simulations, we created sitedirected variants of CA II, derivatized them with a fluorescent label, and examined their response as described below.

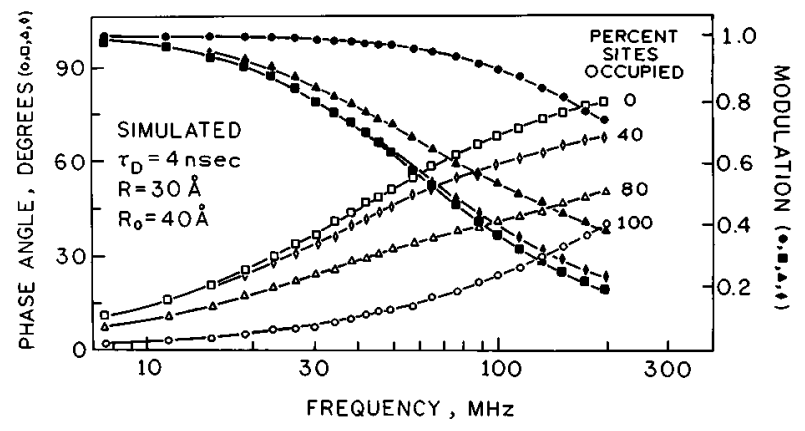

Fig. 3 Simulated phases (open symbols) and modulations (filled symbols) as a function of frequency for a donor fluorophore with a 4-nsec lifetime positioned 30 angstroms from an acceptor binding site; the Forster distance of the donor:acceptor is 40 angstroms. Values are depicted for binding site occupancies of 0 (squares), 40 (diamonds), 80 (triangles), and 100 percent (circles).

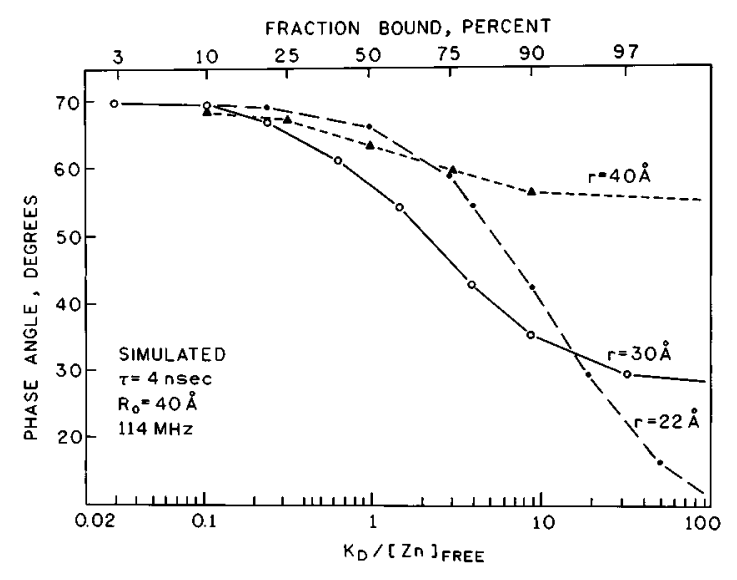

Fig. 4 Simulated phase angles as a function of bound zinc for a 4 -nsec donor at $114 \mathrm{MHz}$ with a 40 -angstrom Forster distance, when the donor is placed 22 angstroms (-0-, filled circles), 30 angstroms (-O-, open circles), or 40 angstroms (- $\mathbf{\Lambda}$, triangles) distant from the acceptor.

\section{EXPERIMENTAL}

Wild type and variants of recombinant human carbonic anhydrase II (H36C, S166C) were constructed, expressed, and purified from Escherichia coli BL21LDE3 as previously described.$^{28}$ The distances from the labeled cysteine to the azosulfamide chromophore (22 angstroms for S166C, 30 angstroms for $\mathrm{H} 36 \mathrm{C}$ ) are estimates based on the crystal structure of unlabeled wild-type enzyme with a different sulfonamide bound to the active site; thus, they are subject to some error. Variants were derivatized with iodoacetamidofluorescein (Molecular Probes, Eugene, $\mathrm{OR}$ ) in the dark at room temperature at $\mathrm{pH}$ 8.0 , after which the reaction was quenched with excess mercaptoethanol and the excess reagent removed by exhaustive dialysis in the cold. Zinc was removed from the enzyme by treatment with aqueous dipicolinate as previously described; ${ }^{29}$ all sub-

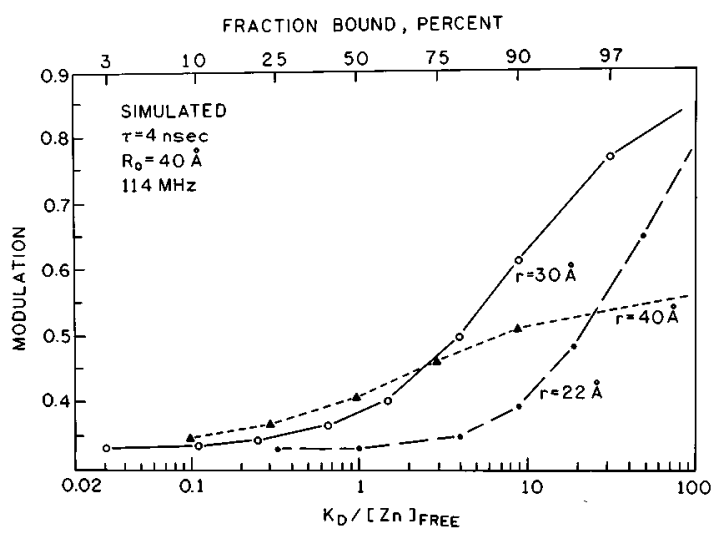

Fig. 5 Simulated modulations as a function of bound zinc for a 4-nsec lifetime donor at $114 \mathrm{MHz}$ with a 40-angstrom Forster distance, when the donor is placed 22 angstroms (-0- , filled circles), 30 angstroms (-O-, open circles), or 40 angstroms (- $\mathbf{\Lambda}$, triangles) away from the acceptor. 


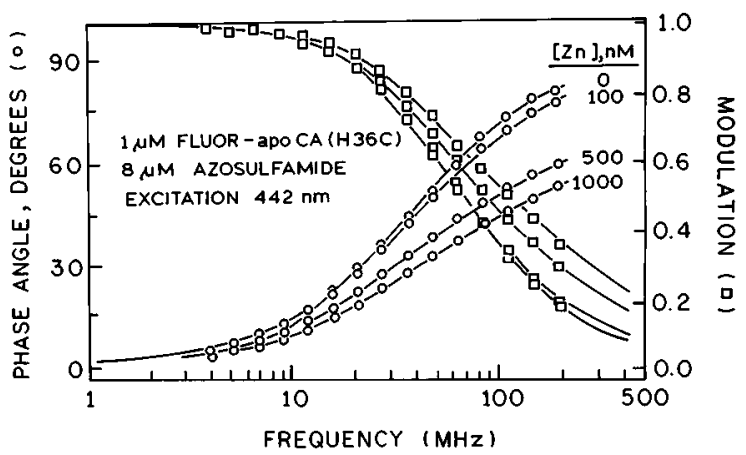

Fig. 6 Frequency-dependent phase shifts (circles) and demodulations (squares) of the $\mathrm{H} 36 \mathrm{C}$ variant of apocarbonic anhydrase labeled with fluorescein $(1 \mu \mathrm{m})$ in the presence of azosulfamide $(8 \mu \mathrm{m})$, as a function of zinc concentration. The lines indicate the best two-component fits to the data.

sequent handling and storage of the enzyme was in plastic, and buffers were treated with chelating resins to prevent metal ion contamination. The HEPES (N-2-hydroxyethyl pipenezine- $N$-2-ethanesulfonic acid) was ultrapure grade from Baker, azosulfamide was from Sigma, and other reagents were A.C.S. reagent grade or better.

Phase fluorometry was performed with a Liconix $4214 \mathrm{NB}$ HeCd laser emitting $12 \mathrm{~mW}$ at $442 \mathrm{~nm}$ as an excitation source, as previously described. ${ }^{30}$ Note that under the circumstances of these experiments the zinc:enzyme binding reaction occurs under conditions Weber has termed the "stoichiometric regime": ${ }^{31}$ the apoenzyme and zinc are both at concentrations manyfold above the equilibrium constant, so that zinc ion will tend to bind quantitatively. To examine the response of the system, an excess of the apoenzyme is present so that we can easily collect data on the (fractionally saturated) system; the binding of zinc to the wild-type enzyme is so tight that fractional saturation can only be achieved at inconveniently low enzyme concentrations.

\section{RESUltS}

We constructed variants of human carbonic anhydrase II with cysteine residues substituted at positions 36 and 166 in the polypeptide chain, which could be specifically labeled with iodoacetamido derivatives of fluorescein, and measured their frequency-dependent phase shifts and demodulations in the presence of excess azosulfamide over a range of zinc concentrations. Data for the H36C variant (30 angstroms from the active site, Figure 2) are shown in Figure 6. As expected, these data are well approximated by two component fits (see below). Compared with the simulations meant to model this system closely (Figure 3), the data are not too similar: note the difference in the phase and modulation curves at saturation with zinc. Phase angles and demodulations at a single frequency as a

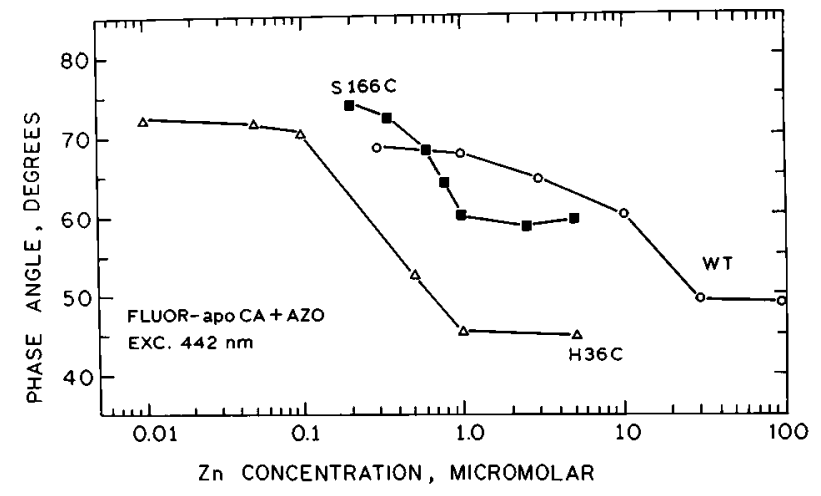

Fig. 7 Phase angles measured for fluoresceinated apocarbonic anhydrases as a function of zinc concentration. Data are depicted for wild type (circles: $5 \mu \mathrm{m}$ enzyme with $5 \mu \mathrm{m}$ azosulfamide at $138 \mathrm{MHz}$ ), S166C (squares: $1 \mu \mathrm{m}$ enzyme with $5 \mu \mathrm{m}$ azosulfamide, $124 \mathrm{MHz}, 22$ angstroms distance), and H36C (triangles: $1 \mu \mathrm{m}$ with $8 \mu \mathrm{m}$ azosulfamide, $114 \mathrm{MHz}, 30$ angstroms distance).

function of zinc concentration for the $\mathrm{H} 36 \mathrm{C}$ and S166C variants, as well as the (randomly derivatized) wild-type enzyme ${ }^{19}$ are depicted in Figures 7 and 8 , respectively.

Several conclusions may be drawn from these data. First, the H36C variant gives a better response than the wild type. The phase-angle difference between zinc-free and zinc-saturated enzyme is 29 degrees for H36C versus 19 degrees for the wild type. Although the response is half maximal at different zinc concentrations for each variant, this is artifactual since the experiments were carried out under slightly varying conditions of enzyme concentration and modulation frequency; from these data we cannot infer any change in the zinc binding constant for the variants. The response for the wild type seems to extend over a broader concentration range of zinc than the variants, which we attribute

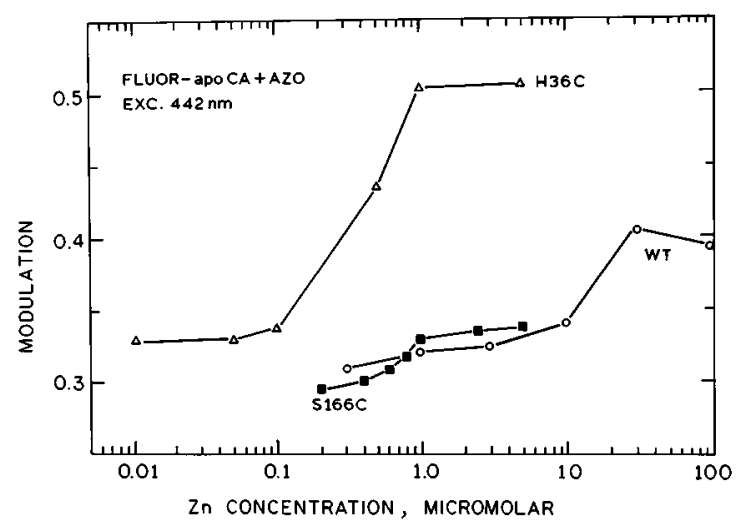

Fig. 8 Modulation ratios measured for fluoresceinated apocarbonic anhydrases as a function of zinc concentration. Data are depicted for wild type (circles: $5 \mu \mathrm{m}$ enzyme with $5 \mu \mathrm{m}$ azosulfamide at $138 \mathrm{MHz}$ ), S166C (squares: $1 \mu \mathrm{m}$ enzyme with $5 \mu \mathrm{m}$ azosulfamide, $124 \mathrm{MHz}, 22$ angstroms distance), and $\mathrm{H} 36 \mathrm{C}$ (triangles: $1 \mu \mathrm{m}$ with $8 \mu \mathrm{m}$ azosulfamide, $114 \mathrm{MHz}, 30$ angstroms distance). 
to the likely heterogeneity of this preparation with respect to sites modified.

The data for the H36C mutant (Figure 6) differ from the simulated data intended to closely model them (Figure 3). Comparison of the frequencydependent data from the variant with the simulation shows that the data differ more near saturation; in particular, the data at saturation for the H36C variant are not well fit by a single exponential of approximately $600 \mathrm{psec}$, which is expected from the theory. Rather, the best fit yields $\tau_{1}$ $=3.725 \pm 0.050 \mathrm{nsec}$ and a fractional intensity of 65 $\pm 0.5 \% ; \tau_{2}=699 \pm 13$ psec with $35 \%$ fractional intensity; and $\chi^{2}=2.3$. Knowing the lifetimes and fractional intensities, we can calculate the proportion of the emitters represented by the bound and free forms (pre-exponential factors). Thus only $75 \%$ of the binding sites appear to be saturated although there is a fivefold molar excess of both zinc and azosulfamide over enzyme, and both concentrations are also well above their respective binding constants. Thus the phase-angle difference between zinc-free and saturated is not as great (29 degrees at $114 \mathrm{MHz}$, Figure 7) as had been predicted (44 degrees, Figure 4). In some ways the model does not accurately reflect the behavior of our system, in that the simulation program assumes the donor is undergoing rapid rotational diffusion with respect to the acceptor. In our case, high measured anisotropies of the conjugated donor (results not shown) and the mode of binding of the azosulfamide lead us to suspect that neither one is rotating rapidly, and thus the efficiency of energy transfer may differ from that suggested by the $R_{0}$ value based on rapid reorientation. However, the recovered lifetime for the bound form $(699 \mathrm{psec})$ is reasonably close to the modeled value (0.6 nsec), suggesting that the energy transfer efficiency cannot differ too much.

The data for the S166C variant (22-angstrom distance) differ even more dramatically from our expectations. In particular, the maximal phase-angle difference is much less (21.4 degrees at $220 \mathrm{MHz}$, Figure 4) than anticipated based on the model (69 degrees at $200 \mathrm{MHz}$, Figure 7). The recovered values from a two-component fit to the frequencydependent data obtained for a saturated system $\left(\tau_{1}=4.02 \pm 0.05 \mathrm{nsec}, f_{1}=0.902 \pm 0.003 ; \tau_{2}\right.$ $=358 \pm 25$ psec, $f_{2}=0.098 ; \chi^{2}=3.8$ ) show that only about $55 \%$ of the labeled protein molecules are exhibiting energy transfer when the system is saturated with zinc and azosulfamide. Again, the recovered quenched lifetime corresponds to the value expected for this $R_{0}$, and thus the efficiency of the energy transfer of the bound form is as expected.

For both enzyme variants, similar arguments may be advanced to explain the variance with the modeled results. In both cases what seems to be occurring is not a decreased efficiency of energy transfer, as judged from the close to expected quenched lifetimes, but rather a partial failure of energy transfer under conditions where it should be nearly quantitative. Three possible reasons may be advanced for this: (1) the zinc affinity has been reduced; (2) the azosulfamide affinity has been reduced; or (3) the protein structure is sufficiently disrupted by removal of zinc and derivatization (or the conditions required) that for some fraction of the protein at least the active site is no longer close to the donor. In view of the wide practice of attaching oligohistidine tails to recombinant proteins as a purification approach, it seems that the active site histidines would retain the capability to bind zinc even with a somewhat disrupted protein; note that the zinc concentration used is six orders of magnitude higher than the binding constant. More likely is the perturbation of the azosulfamide binding affinity, the equilibrium constant of which is only an order of magnitude lower than the concentrations used here. ${ }^{32}$ Because of the substantial inner filter effect at the excitation wavelength, it is difficult to get good quality data at significantly higher azosulfamide concentrations. Also more likely is substantial disruption of the variant protein structure following zinc removal and fluorescent labeling. We have noted with other variants (results not shown) a propensity to aggregate (as judged by fluorescence anisotropy) or precipitate when derivatized with certain fluorescent reagents. While the wild-type apoprotein seems to be stable for months when refrigerated in solution and at least one variant (H94C) is stable enough to be crystallized, ${ }^{33}$ some variants that have been constructed are so unstable that the gene product cannot be isolated as a holoprotein. At the present state of the art, it is difficult to predict which variants will be stable as fluorescent-labeled apoproteins. Fortunately, we can proceed empirically with a given variant and simply measure its response under circumstances where we believe it to be saturated and compare that with the expected result, as we have done here. Finally, efforts are now under way to accurately model the energy transfer efficiency of systems where the donor and acceptor have a single, nonvarying orientation.

In conclusion, we have demonstrated the utility of being able to site-specifically initiate mutagenesis of the biosensor transducer protein carbonic anhydrase for the purpose of measuring zinc concentrations. It should be evident that controlled placement of donors and acceptors is a useful capability for any energy transfer (or other proximitydependent) transduction scheme. Thus, for immunoassays based on energy transfer, ${ }^{22,34}$ the results would clearly be improved if the donors were placed at defined sites on the antibodies and antigens (for competitive assays), or on both antibodies (for sandwich assays). The larger size of the antibody molecule and the complex(es) it makes with antigens (particularly macromolecules) makes it less likely that a donor will be a suitable $\left(0.8 R_{0}\right)$ distance from an acceptor. For instance, donors and 
acceptors on two antibodies participating in a sandwich (immunoradiometric) assay might easily be more than 100 angstroms apart, and even with an excellent spectral overlap, energy transfer is likely to be poor.

A second advantage of great practical value of site-specific mutagenesis is that our fluorescentlabeled enzyme is rather homogeneous. By comparison, most fluorescent protein conjugates are rather ill defined and are likely to vary from batch to batch. This variability requires that lots must be individually tested and recalibrated for use in medical and forensic applications, which is expensive and labor intensive. By comparison, a homogeneous, reproducible preparation would minimize the need for recalibration with each new lot of reagent and therefore the cost of such recalibration. The increased availability of expressed recombinant recognition molecules such as antibodies makes the site-directed mutagenesis we have done a much more viable prospect.

\section{Acknowledgments}

The authors would like to thank the Office of Naval Research for support and their scientific officer, Dr. Harold Bright, for many fruitful discussions; Dr. Joseph Lakowicz and his colleagues at the Center for Fluorescence Spectroscopy at UMAB for the use of the program GAUDIS; Gang Hu for help in preparing the carbonic anhydrase variants, and David Christianson and Chip Lesburg of the University of Pennsylvania for Figure 2 and helpful suggestions. Some of these data were presented in preliminary form at the Seventh International Conference on Chemical, Biochemical, and Environmental Fiber Optic Sensors.

\section{REFERENCES}

1. R.B. Thompson and D.R. Walt, "Emerging strategies for molecular biosensors," ONR Research Rev. 46 (3), 19-29 (1994).

2. O.S. Wolfbeis, Ed., Fiber Optic Chemical Sensors and Biosensors, CRC Press, Boca Raton, FL (1991).

3. D.L. Wise and L. Wingard, Eds. Biosensors with Fiber Optics, Humana Press, Clifton, NJ (1991).

4. J.R. Lakowicz and R.B. Thompson, Eds. Advances in Fluorescence Sensing Technology, Proc. SPIE 1885 (1993).

5. J.R. Lakowicz, Ed. Advances in Fluorescence Sensing Technology II Proc. SPIE 2388 (1995).

6. R.B. Thompson and E.R. Jones, "Enzyme-based fiber optic zinc biosensor," Anal. Chem. 65, 730-734 (1993).

7. L.C. Shriver-Lake, K.A. Breslin, P.T. Charles, D.W. Conrad, J.P. Golden, and F.S. Ligler, "Detection of TNT in water using an evanescent wave biosensor," Anal. Chem. 67, 24312435 (1995).

8. R.A. Ogert, J.E. Brown, B.R. Singh, L.C. Shriver-Lake, and F.S. Ligler, "Detection of Clostridium botulinum toxin A using a fiber optic-based biosensor," Anal. Biochem. 205, 306-312 (1992).

9. B.J. Tromberg, M.J. Sepaniak, T. Vo-Dinh, and G.D. Griffin, "Fiber optic chemical sensors for competitive-binding fluoroimmunoassay," Anal. Chem. 59, 1226-1230 (1987).

10. L.A. Saari and W.R. Seitz, "Immobilized morin as a fluorescence sensor for Al(III)," Anal. Chem. 55, 667-670 (1983).

11. P.Yager, R.B. Thompson, and S. Merlo, "Fluorescence-based optical sensor and method for detection of lipid-soluble analytes," U.S. Patent No. 5,094,819 (1992).
12. M.E. Lippitsch, J. Pusterhofer, M.J.P. Leiner, and O.S. Wolfbeis, "Fibre-optic oxygen sensor with the fluorescence decay time as the information carrier," Anal. Chim. Acta 207, 1-6 (1988).

13. J.R. Lakowicz, "Fluorescence lifetime-based sensing," Laser Focus World 28 (5), 127 (1992).

14. H. Szmacinski and J.R. Lakowicz, "Lifetime-based sensing," in Topics in Fluorescence Spectroscopy, Vol. 4: Probe Design and Chemical Sensing, J.R. Lakowicz, Ed., pp. 295-334, Plenum Press, New York (1994).

15. H. Szmacinski and J.R. Lakowicz, "Optical measurements of $\mathrm{pH}$ using fluorescence lifetimes and phase-modulation fluorometry," Anal. Chem. 65, 1668-1674 (1993).

16. R.B. Thompson and M.W. Patchan, "Fluorescence lifetimebased biosensing of zinc: Origin of the broad dynamic range," J. Fluoresc. 5, 123-130 (1995).

17. R.B. Thompson, "Fluorescence-based fiber optic sensors," in Topics in Fluorescence Spectroscopy, Vol. 2: Principles, J.R. Lakowicz, Ed., pp. 345-365, Plenum Press, New York (1991).

18. J.R. Lakowicz, H. Szmacinski, and R.B. Thompson, "Fluorescence lifetime-based sensing: Applications to clinical chemistry and cellular imaging," in Ultrasensitive Laboratory Diagnostics, G.E. Cohn, Ed., Proc. SPIE 1895, 2-17 (1993).

19. R.B. Thompson and M.W. Patchan, "Lifetime-based fluorescence energy transfer biosensing of zinc," Anal. Biochem. 227, 123-128 (1995).

20. R.B. Thompson, Z. Ge, M.W. Patchan, and C.A. Fierke, "Energy transfer-based fiber optic metal ion biosensor," $A d-$ vances in Fluorescence Sensing Technology, J.R. Lakowicz, Ed., Proc. SPIE 2388, 138-147 (1995).

21. J. Sipior, S. Bambot, M. Roumauld, G.M. Carter, J.R. Lakowicz, and G. Rao, "A lifetime-based optical $\mathrm{CO}_{2}$ gas sensor with blue or red excitation and Stokes or anti-Stokes detection," Anal. Chem. 227, 309-318 (1995).

22. J.R. Lakowicz, B.P. Maliwal, A. Ozinskas, and R.B. Thompson, "Fluorescence lifetime energy-transfer immunoassay quantified by phase-modulation fluorometry," Sensors and Actuators B 12, 65-70 (1993).

23. C.E. White and R.J. Argauer, Fluorescence Analysis: A Practical Approach, Marcel Dekker, New York (1970).

24. R.D. Spencer and G. Weber, "Cross-correlation phase fluorometer," Ann. N.Y. Acad. Sci. 158, 361-376 (1969).

25. J.R. Lakowicz and I. Gryczynski, "Frequency-domain fluorescence spectroscopy," in Topics in Fluorescence Spectroscopy Vol. 1: Techniques, J.R. Lakowicz, Ed., Chapter 5, pp. 293-335, Plenum Press, New York (1991).

26. J.E. Coleman, "Carbonic anhydrase-azosulfonamide complexes. Optical rotatory dispersion and circular dichroism," J. Am. Chem. Soc. 89, 6757-6759 (1967).

27. J.R. Lakowicz, I. Gryczynski, W. Wiczk, J. Kusba, and M.L. Johnson, "Correction for incomplete labeling in the measurement of distance distributions by frequency-domain fluorometry," Anal. Biochem. 195, 243-254 (1991).

28. J.F. Krebs, F. Rana, R.A. Dluhy, and C.A. Fierke, "Kinetic and spectroscopic studies of hydrophilic amino acid substitutions in the hydrophobic pocket of human carbonic anhydrase II, Biochemistry 32, 9896-9900 (1993).

29. J.B. Hunt, M.-J. Rhee, and C.B. Storm, "A rapid and convenient preparation of apocarbonic anhydrase," Anal. Biochem. 79, 614-617 (1977).

30. R.B. Thompson and E. Gratton, "Phase fluorimetric method for determination of standard lifetimes," Anal. Chem. 60, 670-674 (1988).

31. D. Davenport, "Use of fluorescence in binding studies," in Fluorescence Spectroscopy: An Introduction for Biology and Medicine, A.J. Pesce, C.-G. Rosen, and T.L. Pasby, Eds., pp. 203-240, Marcel Dekker, New York (1971).

32. H.A. Krebs, Biophys. J. 43, 525-528 (1948).

33. R.S. Alexander, L.L. Kiefer, C.A. Fierke, and D.W. Christianson, "Engineering the zinc binding site of human carbonic anhydrase II: Structure of the his-94 to cys apoenzyme in a new crystalline form," Biochemistry 32, 1510-1518 (1993).

34. E.F. Ullman and M. Schwarzberg, "Fluorescence quenching with immunological pairs in immunoassays," U.S. Patent No. 4,261,968 (1981). 